\title{
Prototipo de simulador dental para mejorar la microfiltración en las restauraciones de las piezas dentarias
}

\section{Prototype of dental pretender to improve the microfiltering in the restorations of the dentalpieces}

\author{
Víctor Orlando Machuca Reyes*
}

http://dx.doi.org/10.21503/CienciayDesarrollo.2007.v8.03

\section{RESUMEN}

El objetivo de la investigación ha sido el diseño y elaboración de un prototipo simulador de contactos proximales para mejorar la microfilmación en las restauraciones de piezas dentarias.

La unidad de análisis la constituyen cincuenta odontólogos que trabajan en tres policlínicos periféricos de la Sanidad de la PNP, y el diseño metodológico empleado ha sido de tipo aplicativo y de nivel descriptivoexplicativo y correlacional. Se aplicaron encuestas de opinión, así como la observación directa y la revisión documental valiéndose de técnicas de recopilación de datos.

La conclusión general fue que existe suficiente evidencia científica para afirmar que el prototipo simulador dental mejorará las microfiltraciones en las restauraciones de las piezas dentarias de los pacientes que acuden a los consultorios externos de los tres policlínicos periféricos de la Sanidad de la Policía Nacional del Perú en Lima Metropolitana.

Palabras clave: prototipo, simulador dental, microfiltración, restauración de piezas dentarias, odontología.

\section{ABSTRACT}

The Aim (Lens) of the Investigation has been the design and production of a prototype malingerer of contacts proximales to improve the microfilming in the restorations of pieces dentarias, inside the practice odontológica.

The unit of analysis they constitute the Odontologists in number of 50 that are employed at three Polyclinical peripheral of the Health of the PNP and with regard to the methodological design employee has been of type Aplicativo and of Descriptive - explanatory level and Correlacional. I apply public-opinion polls to him (you, them), as well as the direct observation and the documentary review as technologies (skills) of summary of information.

The General Conclusion was sufficient scientific evidence Exists to affirm that the prototype dental malingerer they will improve the microfiltrations in the restorations of the pieces dentarias, of the patients who come to the external doctor's offices of the Polyclinical peripheral three of the Health of the National Police of the Peru in Metropolitan Lima.

Key words: prototype, dental malingerer, microfiltration, restoration of the pieces dentarias, odontology.

* Magíster por la Escuela de Postgrado de la Universidad Alas Peruanas. 


\section{INTRODUCCIÓN}

En la actualidad, el avance tan acelerado en biomateriales dentales, específicamente de resinas compuestas, exige al facultativo tener la oportunidad de validar sus conocimientos en prácticas odontológicas más sencillas, eficientes y con múltiples opciones para tratamientos cada vez más exigentes.

El desarrollo de la tecnología adhesiva en la especialidad de odontología estética restauradora está atravesando por un momento de desarrollo acelerado, evidenciado por la aparición de un sistema adhesivo altamente competente. Sin embargo, debemos tener presente que aún existen ciertas limitaciones en la parte clínica, como son la insuficiente adhesión a la dentina y el estrés superficial residual de las resinas polimerizadas.

El manejo clínico de polimerización de los materiales de aplicación directa, como es el caso de las resinas compuestas, se encuentra en su máxima evolución tecnológica. En la actualidad se vienen utilizando diferentes técnicas, como la de laminación vertical (de aplicación gradual), la de aplicación directa y la de laminación horizontal, que tienen como objetivo principal reducir el estrés generado por la contracción de polimerización. Esta reacción trae como consecuencia que la resina compuesta no fatigue el sistema adhesivo y no se produzcan brechas importantes, mejorando así la longevidad de la restauración.

\section{MATERIAL Y MÉTODO}

El modelo de investigación seguido ha sido el propuesto por el Dr. Jorge Lazo Arrasco en su texto Pedagogía universitaria, editado por la Universidad Alas Peruanas el año 2006.

\section{Conceptualización y operacionalización de} variables

Variable dependiente: Microfiltración en las restauraciones de las piezas dentarias.

CONCEPTO : Pasajes de bacterias, sustancias fluidas, químicas, iones y moléculas entre el diente y el material de restauración.

INDICADOR : Brechas en los márgenes de las restauraciones.

ESCALA : Nominal.

Variable independiente: Polimerización.

CONCEPTO : Grado de conversión de resina fotopolimerizable.

INDICADOR : Dureza de polimerización.

Variable independiente: Material de restauración.

CONCEPTO : Material de diferentes estructuras inocuas a los tejidos dentales

INDICADOR : Estéticas y rígidas, compactas.

Variable independiente: Simulador dental.

CONCEPTO : Prototipo de simulador de contactos proximales que permiten disminuir la microfiltración.

INDICADOR : Calibración de alta precisión de los contactos proximales.

\section{Tipo de investigación}

Por la forma en que se ha planteado y por los objetivos de la misma, esta investigación reúne las características de ser aplicada, en razón de que para llevarla a cabo se utilizaron conocimientos necesarios para "diseñar un prototipo 
de simulador dental" y aplicarlo en "mejorar la microfiltración de las restauraciones en las piezas dentarias".

\section{Nivel de investigación}

Esta investigación reúne las condiciones para ser considerada como descriptiva, correlacional y explicativa.

Es descriptiva porque en un primer momento se describirá cómo el diseño del prototipo de simulador dental mejorara la microfiltración en las restauraciones de las piezas dentarias. Es correlacional debido a la relación que existe entre las variables materia de la investigación. Y es explicativa porque explica de manera clara y comprensible el uso del diseño del prototipo de simulador dental.

\section{Método de la investigación}

Explicativo, en razón de que el tipo de relación que existe entre la microfilmación de las restauraciones dentales y el diseño del prototipo dental es de causa-efecto.

\section{Diseño de la investigación}

\section{Universo}

Lo constituyen los odontólogos que laboran en los hospitales y policlínicos de las Fuerzas Armadas con sede en Lima.

\section{Técnica e instrumentos y fuentes de recolección de datos}

Se han aplicado las siguientes técnicas:

a. La revisión documental efectuada en libros de la especialidad, artículos de revistas e Internet, empleando fichas de registro, resúmenes y comentarios críticos.

b. La observación directa.

c. La encuesta, aplicada a los odontólogos.

\section{Población}

La población de estudio estará constituido por los odontólogos que laboran en los tres policlínicos periféricos de la Sanidad PNP, como son el Policlínico San Diego (Norte), el Policlínico Callao PNP (Este) y el Policlínico Geriátrico PNP (Sur).

La población total que será estudiada son 50 odontólogos que laboran en dichos policlínicos.

\section{Muestra}

Considerando el número de odontólogos, no se considera muestra alguna, pues se ha trabajado con el total de ellos.

\section{Procesamiento de datos}

En un primer momento se procedió a la codificación y tabulación de los datos obtenidos, así como a la elaboración de gráficos. Posteriormente, con la ayuda del programa estadístico electrónico SPSS-12, se procedió a vaciar la información en las tablas o cuadros correspondientes.

A continuación se siguió con el análisis de los datos y la interpretación correspondiente, teniendo en cuenta las hipótesis planteadas.

\section{RESULTADOS DE LA INVESTIGACIÓN}

Resultados de la aplicación de la encuesta

P1) ¿Cuál es su estado civil?

Estado civil

\begin{tabular}{clcc} 
& & Frecuencia & Porcentaje \\
\hline Válidos & Soltero & 18 & 36,0 \\
& Casado & 27 & 54,0 \\
& Concubinato & 5 & 10,0 \\
& Total & 50 & 100,0 \\
\hline
\end{tabular}

El 54\% de los encuestados es casado, el 36\% soltero y el $10 \%$ está en situación de concubinato. 
P2) ¿Cuántos años tiene Ud.?

\section{Edad}

\section{Frecuencia Porcentaje}

\begin{tabular}{|llrr|}
\hline Válidos & $22-30$ & 22 & 44,0 \\
& $31-50$ & 23 & 46,0 \\
\hline & 51 - más & 5 & 10,0 \\
& Total & 50 & 100,0 \\
\hline
\end{tabular}

El rango de edad mayoritario está entre los 31 y 50 años, en la plenitud de sus responsabilidades profesionales.

P3) ¿Quésexo posee?

\section{Sexo}

\section{Frecuencia Porcentaje}

\begin{tabular}{llrr}
\hline Válidos & F & 24 & 48,0 \\
& M & 26 & 52,0 \\
& Total & 50 & 100,0 \\
\hline
\end{tabular}

El $52 \%$ son varones y el $48 \%$ son mujeres.

P4) ¿Qué tiempo ejerce la profesión?

Tiempo que ejerce la profesión

\section{Frecuencia Porcentaje}

\begin{tabular}{llrr}
\hline Válidos & 2 - 7 años & 22 & 44,0 \\
& 8 - 12 años & 23 & 46,0 \\
\hline 12 - más & 5 & 10,0 \\
\hline Total & 50 & 100,0 \\
\hline
\end{tabular}

El 46\% la ejerce entre 8 a 12 años, mientras que el $10 \%$ lo hace de 12 años a más.

P5) ¿En quépoliclinico trabaja?

\begin{tabular}{lcc}
\multicolumn{2}{c}{ Policlínico } & \\
& Frecuencia & Porcentaje \\
\hline Policlínico Callao PNP & 19 & 38,0 \\
\hline Policlínico San Diego PNP & 17 & 34,0 \\
Policlínico Geriátrico PNP & 14 & 28,0 \\
Total & 50 & 100,0 \\
\hline
\end{tabular}

El 38\% trabaja en el Policlínico de la Policía Nacional del Callao, mientras que el 28\% lo hace en el Policlínico Geriátrico de la Policía Nacional.

P6) ¿Las restauraciones siempre quedan bien adaptadas?

Las restauraciones siempre quedan bien adaptadas

\section{Frecuencia Porcentaje}

\begin{tabular}{llrr}
\hline Válidos & No & 14 & 28,0 \\
& Sí & 36 & 72,0 \\
& Total & 50 & 100,0 \\
\hline
\end{tabular}

El $72 \%$ opinó que sí, mientras el 28\% opinó que no.

P7) ¿Las restauraciones grandes o complejas presentan mala adaptación?

¿Las restauraciones grandes o complejas presentan mala adaptación?

Frecuencia Porcentaje

\begin{tabular}{lllr}
\hline Válidos & No & 26 & 52,0 \\
& Sí & 24 & 48,0 \\
& Total & 50 & 100,0 \\
\hline
\end{tabular}

El 48\% opinó que sí, mientras el 52\% opinó que no.

P8) ¿Las grietas que se observan en las restauraciones conllevan a un mal pronóstico de la adaptación?

¿Las grietas que se observan en las restauraciones conllevan a un mal pronóstico de la adaptación?

Frecuencia Porcentaje

\begin{tabular}{llrr}
\hline Válidos & No & 23 & 46,0 \\
& Sí & 27 & 54,0 \\
& Total & 50 & 100,0 \\
\hline
\end{tabular}

El 54\% opinó que sí, mientras el 46\% opinó que no. 
P9) ¿Ud.cree que el odontólogo es la causa directa de la mala adaptación de las restauraciones?

¿Usted cree que el odontólogo es la causa directa de la mala adaptación de las restauraciones?

\section{Frecuencia Porcentaje}

\begin{tabular}{lllr}
\hline Válidos & No & 21 & 42,0 \\
& Sí & 29 & 58,0 \\
& Total & 50 & 100,0 \\
\hline
\end{tabular}

El 58\% opinó que sí, mientras el 42\%, que no.

P10) ¿Ud. cree que por falta de tecnologia e instrumentos no se puede mejorar una mala adaptación?

¿Usted cree que por falta de tecnología e instrumentos no se puede mejorar una mala adaptación?

\section{Frecuencia Porcentaje}

\begin{tabular}{llrr}
\hline Válidos & No & 20 & 40,0 \\
& Sí & 30 & 60,0 \\
& Total & 50 & 100,0 \\
\hline
\end{tabular}

El 60\% opinó que sí, mientras el 40\%, que no.

\section{P11) ¿Ud. cree que existe la técnica adecuada para desaparecer la mala adaptación marginal?}

¿Usted cree que existe la técnica adecuada para desaparecer la mala adaptación marginal?

\section{Frecuencia Porcentaje}

\begin{tabular}{llrr}
\hline Válidos & No & 18 & 36,0 \\
& Sí & 32 & 64,0 \\
& Total & 50 & 100,0 \\
\hline
\end{tabular}

El 64\% opinó que sí, mientras el 36\%, que no.

P12) ¿Sabe cómo disminuir la contracción de polimerización de estos materiales?

¿Sabe cómo disminuir la contracción de polimerización de estos materiales?

\section{Frecuencia Porcentaje}

\begin{tabular}{llrr}
\hline Válidos & No & 25 & 50,0 \\
& Sí & 25 & 50,0 \\
& Total & 50 & 100,0 \\
\hline
\end{tabular}

El 50\% opinó que sí, mientras el otro 50\%, que no.
P13) ¿Utiliza las técnicas adecuadas para insertar materiales en restauraciones grandes?

¿Utiliza las técnicas adecuadas para insertar materiales en restauraciones grandes?

\section{Frecuencia Porcentaje}

\begin{tabular}{llrr}
\hline Válidos & No & 17 & 34,0 \\
& Sí & 33 & 66,0 \\
& Total & 50 & 100,0 \\
\hline
\end{tabular}

El 66\% opinó que sí, mientras el 34\%, que no.

P14) ¿Utiliza los aditamentos o aparatos adecuados para insertar material en restauraciones complejas y disminuir la microfiltración?

¿Utiliza los aditamentos o aparatos adecuados para insertar material en restauraciones complejas y disminuir la microfiltración?

Frecuencia Porcentaje

\begin{tabular}{llrr}
\hline Válidos & No & 18 & 36,0 \\
& Sí & 32 & 64,0 \\
& Total & 50 & 100,0 \\
\hline
\end{tabular}

El 64\% opinó que sí, mientras el 35\%, que no.

P15) ¿En cavidades complejas siempre existe el riesgo de microfiltración?

¿En cavidades complejas siempre existe el riesgo de microfiltraciòn?

Frecuencia Porcentaje

\begin{tabular}{lllr}
\hline Válidos & No & 17 & 34,0 \\
& Sí & 33 & 66,0 \\
& Total & 50 & 100,0 \\
\hline
\end{tabular}

El 66\% opinó que sí, mientras el 34\%, que no.

P16) ¿Utiliza limadura de plata en restauraciones posteriores?

¿Utiliza limadura de plata en restauraciones posteriores?

\begin{tabular}{llcc} 
& & Frecuencia & Porcentaje \\
\hline Válidos & No & 26 & 52,0 \\
& Sí & 24 & 48,0 \\
& Total & 50 & 100,0 \\
\hline
\end{tabular}

El 52\% opinó que no, mientras el 48\%, que sí. 
P17) ¿Utiliza con frecuencia material restaurador fotopolimerizable en el sector posterior?

¿Utiliza con frecuencia material restaurador fotopolimerizable en el sector posterior?

\section{Frecuencia Porcentaje}

\begin{tabular}{lllr}
\hline Válidos & No & 15 & 30,0 \\
& Sí & 35 & 70,0 \\
& Total & 50 & 100,0 \\
\hline
\end{tabular}

El 70\% opinó que sí, mientras el 30\%, que no.

\section{P18) ¿Utiliza siempre base en cavidades profundas?}

¿Utiliza siempre base en cavidades profundas?

\section{Frecuencia Porcentaje}

\begin{tabular}{llrr}
\hline Válidos & No & 26 & 52,0 \\
& Sí & 24 & 48,0 \\
& Total & 50 & 100,0 \\
\hline
\end{tabular}

El 52\% opinó que no, mientras el 48\%, que sí.

\section{P19) ¿Tiene dificultad para llevar la fuente de luz hacia el material?}

¿Tiene dificultad para llevar la fuente de luz hacia el material?

\section{Frecuencia Porcentaje}

\begin{tabular}{llrr}
\hline Válidos & No & 25 & 50,0 \\
& Sí & 25 & 50,0 \\
& Total & 50 & 100,0 \\
\hline
\end{tabular}

El 50\% opinó que sí, mientras el otro $50 \%$ opinó que no.

P20) ¿Utiliza otros aditamentos para disminuir la distancia de la punta de la fuente de luz al material fotocurado?

¿Utiliza otros aditamentos para disminuir la distancia de la punta de la fuente de luz al material fotocurado?

\section{Frecuencia Porcentaje}

\begin{tabular}{llrr}
\hline Válidos & No & 23 & 46,0 \\
& Sí & 27 & 54,0 \\
& Total & 50 & 100,0 \\
\hline
\end{tabular}

El 54\% opinó que sí, mientras el 46\%, que no.
P21) ¿Conoce usted un prototipo dental de simulador de contactos proximales que sirva para realizar estudios in vitro?

¿Conoce usted un prototipo dental de simulador de contactos proximales que sirva para realizar estudios in vitro?

\section{Frecuencia Porcentaje}

\begin{tabular}{rlrr}
\hline Válidos & No & 19 & 38,0 \\
& Sí & 31 & 62,0 \\
& Total & 50 & 100,0 \\
\hline
\end{tabular}

El 62\% opinó que sí, mientras el 38\%, que no.

P22) Si existiera, ¿usted cree que ayudaria para mejorar su labor en las microfiltraciones dentales?

Si existiera, ¿usted cree que ayudaría para mejorar su labor en las microfiltraciones dentales?

Frecuencia Porcentaje

\begin{tabular}{llrr}
\hline Válidos & No & 18 & 36,0 \\
& Sí & 32 & 64,0 \\
& Total & 50 & 100,0 \\
\hline
\end{tabular}

El 64\% opinó que sí, mientras el 36\%, que no.

P23) ¿El prototipo que sirva para medir de simulador de contactos proximales ayudaría con la ciencia de los materiales fotopolimerizables?

¿El prototipo que sirva para medir de simulador de contactos proximales ayudaría con la de contactos proximales ayudaría con la

\section{Frecuencia Porcentaje}

\begin{tabular}{llrr}
\hline Válidos & No & 22 & 44,0 \\
& Sí & 28 & 56,0 \\
& Total & 50 & 100,0 \\
\hline
\end{tabular}

El 56\% opinó que sí, mientras el 44\%, que no.

\section{P24) ¿Usted cree que es costoso diseñar un prototi-} po para este propósito?

\section{¿Usted cree que es costoso diseñar un} prototipo para este propósito?

Frecuencia Porcentaje

\begin{tabular}{llrr}
\hline Válidos & No & 25 & 50,0 \\
& Sí & 25 & 50,0 \\
& Total & 50 & 100,0 \\
\hline
\end{tabular}


El 50\% opinó que sí, mientras el otro 50\% opinó que no.

\section{Empleo del modelo estadístico}

El modelo estadístico empleado guarda relación con la metodología científica para la recolección, organización, tabulación, presentación y análisis de datos. Esto nos ha permitido planear, analizar e interpretar los resultados de la investigación.

Las operaciones realizadas con los datos obtenidos a través de las diversas técnicas de recopilación de datos (observación directa, revisión documental y encuestas) han sido las siguientes:

$1^{\circ}$. Procesamiento de datos, es decir, la organización de la información que se obtuvo, la misma que ha posibilitado extraer conclusiones de sus análisis.

a. La codificación o asignación de símbolos o números a todas y cada una de las categorías de respuestas recogidas en la información.

b. La tabulación o proceso que permite determinar la frecuencia del fenómeno objeto de investigación.

c. La formación de tablas o cuadros que posibilita la agrupación de los datos en tablas o cuadros de doble entrada, convenientemente preparados, facilitando así la aplicación de métodos estadísticos, especialmente de los elementos de la estadística descriptiva, analizando los estadígrafos de posición o tendencia central, como la media, la mediana y la moda.

\section{Contrastación de hipótesis}

\section{Hipótesis principal}

Ho: Con el prototipo simulador dental no mejorará la microfiltración en las restauraciones de las piezas dentarias.

H1: Con el prototipo simulador dental mejorará la microfiltración en las restauraciones de las piezas dentarias.

Tabla de contingencia. ¿Conoce usted un prototipo dental de simulador de contactos proximales que sirva para realizar estudios in vitro? Si Existiera, ¿usted cree que ayudaría a mejorar su labor en las microfiltraciones dentales?

\begin{tabular}{|c|c|c|c|c|}
\hline \multirow{2}{*}{$\%$ del total } & & $\begin{array}{l}\text { Si existiera } \\
\text { ayudaría a } \\
\text { en la microfil }\end{array}$ & $\begin{array}{l}\text { cree que } \\
\text { su labor } \\
\text { es dentales? }\end{array}$ & \multirow[t]{2}{*}{ Total } \\
\hline & & No & Sí & \\
\hline $\begin{array}{l}\text { ¿Conoce usted un prototipo } \\
\text { dental de simulador de } \\
\text { contactos proximales que }\end{array}$ & No & $28,0 \%$ & $10,0 \%$ & $38,0 \%$ \\
\hline $\begin{array}{l}\text { sirva para realizar estudios } \\
\text { in vitro? }\end{array}$ & Sí & $8,0 \%$ & $54,0 \%$ & $62,0 \%$ \\
\hline Total & & $36,0 \%$ & $64,0 \%$ & $100,0 \%$ \\
\hline
\end{tabular}




\section{Pruebas de Chi-cuadrado}

\begin{tabular}{lccccc} 
& Valor & gl & $\begin{array}{c}\text { Sig. asintótica } \\
\text { (bilateral) }\end{array}$ & $\begin{array}{c}\text { Sig. exacta } \\
\text { (bilateral) }\end{array}$ & $\begin{array}{c}\text { Sig. exacta } \\
\text { (unilateral) }\end{array}$ \\
\hline $\begin{array}{l}\text { Chi-cuadrado de Pearson } \\
\text { Corrección por continuidad }\end{array}$ & $18,889^{\mathrm{b}}$ & 1 & 0,000 & & \\
$\begin{array}{l}\text { Razón de verosimilitud } \\
\text { Estadístico exacto de Fisher }\end{array}$ & 16,343 & 1 & 0,000 & & \\
Asociación lineal por lineal & 19,599 & 1 & 0,000 & & 0,000 \\
$\mathrm{~N}^{0}$ de casos válidos & 18,511 & & & 0,000 & \\
\hline
\end{tabular}

a. Calculado sólo para una tabla de $2 \times 2$.

b. 0 casillas $(0 \%)$ tienen una frecuencia esperada inferior a 5 . La frecuencia mínima esperada es 6,84 .

\begin{tabular}{llcccc} 
& \multicolumn{2}{c}{ Medidas simétricas } & & & \\
& & Valor & $\begin{array}{c}\text { Error tip. }^{2} \\
\text { asint. }^{2}\end{array}$ & $\begin{array}{c}\text { T. aproximada } \\
\text { b }\end{array}$ & $\begin{array}{c}\text { Sig. } \\
\text { aproximada }\end{array}$ \\
\hline Intervalo por intervalo & R de Pearson & 0,615 & 0,116 & 5,398 & $0,000^{c}$ \\
Ordinal por ordinal & Correlación de Spearman & 0,615 & 0,116 & 5,398 & $0,000^{c}$ \\
$N^{0}$ de casos válidos & & 50 & & & \\
\hline
\end{tabular}

a. Asumiendo la hipótesis alternativa.

b. Empleando el error típico asintótico basado en la hipótesis nula.

c. Basada en la aproximación normal.

¿El prototipo que sirva para medir de simulador de contactos proximales ayudaría con la ciencia de los materiales fotopolimerizables? Si existiera, ¿usted cree que ayudaría para mejorar su labor en las microfiltraciones dentales?

Tabla de contingencia.

\begin{tabular}{|c|c|c|c|c|}
\hline \multirow{2}{*}{$\%$ del total } & & $\begin{array}{l}\text { Si existier } \\
\text { ayudaría } \\
\text { en la microf }\end{array}$ & $\begin{array}{l}\text { d cree que } \\
\text { ir su labor } \\
\text { ies dentales? }\end{array}$ & \multirow[t]{2}{*}{ Total } \\
\hline & & No & Sí & \\
\hline $\begin{array}{l}\text { ¿El prototipo que sirva } \\
\text { para medir de simulador de }\end{array}$ & No & $26,0 \%$ & $18,0 \%$ & $44,0 \%$ \\
\hline $\begin{array}{l}\text { contactos proximales ayudaría } \\
\text { con la ciencia de los materiales } \\
\text { fotopolimerizables? }\end{array}$ & Sí & $10,0 \%$ & $46,0 \%$ & $56,0 \%$ \\
\hline Total & & $36,0 \%$ & $64,0 \%$ & $100,0 \%$ \\
\hline
\end{tabular}

Pruebas de Chi-cuadrado

\begin{tabular}{|c|c|c|c|c|c|}
\hline & Valor & gl & $\begin{array}{l}\text { Sig. asintótica } \\
\text { (bilateral) }\end{array}$ & $\begin{array}{l}\text { Sig. exacta } \\
\text { (bilateral) }\end{array}$ & $\begin{array}{l}\text { Sig. exacta } \\
\text { (unilateral) }\end{array}$ \\
\hline Chi-cuadrado de Pearson & $9,091^{\mathrm{b}}$ & 1 & 0,003 & & \\
\hline Corrección por continuidad $^{\mathrm{a}}$ & 7,390 & 1 & 0,007 & & \\
\hline Razón de verosimilitud & 9,298 & 1 & 0,002 & & \\
\hline Estadístico exacto de Fisher & & & & 0,004 & 0,003 \\
\hline Asociación lineal por lineal & 8,910 & 1 & 0,003 & & \\
\hline $\mathrm{N}^{0}$ de casos válidos & 50 & & & & \\
\hline
\end{tabular}




\begin{tabular}{llcccc} 
& \multicolumn{3}{c}{ Medidas simétricas } & & \\
& Valor & $\begin{array}{c}\text { Error tip. } \\
\text { asint. }^{\mathrm{a}}\end{array}$ & $\begin{array}{c}\text { T. aproximada } \\
\text { b }\end{array}$ & $\begin{array}{c}\text { Sig. } \\
\text { aproximada }\end{array}$ \\
\hline Intervalo por intervalo & R de Pearson & 0,426 & 0,129 & 3,266 & $0,002^{\mathrm{c}}$ \\
Ordinal por ordinal & Correlación de Spearman & 0,426 & 0,129 & 3,266 & $0,002^{\mathrm{c}}$ \\
$\mathrm{N}^{0}$ de casos válidos & & 50 & & & \\
\hline
\end{tabular}

a. Asumiendo la hipótesis alternativa.

b. Empleando el error típico asintótico basado en la hipótesis nula.

c. Basada en la aproximación normal.

El p-value es menor a un $\alpha=0,05$, por lo que se rechaza la hipótesis planteada.

Existe suficiente evidencia estadística para afirmar que con el prototipo simulador dental se mejorará la microfiltración en las restauraciones de las piezas dentarias, con un nivel de significación de 0,05 .

\section{Hipótesis secundarias}

Ho: Los factores de la cavidad bucal no serán determinantes en la mejora de la microfiltración de las restauraciones de las piezas dentarias.

H1: Los factores de la cavidad bucal serán determinantes en la mejora de la microfiltración de las restauraciones de las piezas dentarias.

Tabla de contingencia. ¿En cavidades complejas siempre existe el riesgo de microfiltración? ¿Utiliza siempre base en cavidades profundas?

\begin{tabular}{|c|c|c|c|c|}
\hline \multirow[b]{2}{*}{$\%$ del total } & & \multicolumn{2}{|c|}{$\begin{array}{l}\text { ¿Utiliza siempre base } \\
\text { en cavidades profundas? }\end{array}$} & \multirow{2}{*}{ Total } \\
\hline & & No & Sí & \\
\hline ¿En cavidades complejas & No & $30,0 \%$ & $4,0 \%$ & $34,0 \%$ \\
\hline $\begin{array}{l}\text { siempre existe el riesgo de } \\
\text { microfiltración? }\end{array}$ & Sí & $22,0 \%$ & $44,0 \%$ & $66,0 \%$ \\
\hline Total & & $52,0 \%$ & $48,0 \%$ & $100,0 \%$ \\
\hline
\end{tabular}

Pruebas de Chi-cuadrado

\begin{tabular}{lccccc} 
& Valor & gl & $\begin{array}{c}\text { Sig. asintótica } \\
\text { (bilateral) }\end{array}$ & $\begin{array}{c}\text { Sig. exacta } \\
\text { (bilateral) }\end{array}$ & $\begin{array}{c}\text { Sig. exacta } \\
\text { (unilateral) }\end{array}$ \\
\hline Chi-cuadrado de Pearson & $13,550^{\mathrm{b}}$ & 1 & 0,000 & & \\
Corrección por continuidad & 11,439 & 1 & 0,001 & & \\
Razón de verosimilitud & 14,910 & 1 & 0,000 & & \\
Estadístico exacto de Fisher & & & & 0,000 & \\
Asociación lineal por lineal & 13,279 & 1 & 0,000 & & \\
$\mathrm{~N}^{0}$ de casos válidos & 50 & & & & \\
\hline
\end{tabular}

a. Calculado sólo para una tabla de $2 \times 2$.

b. 0 casillas $(0 \%)$ tienen una frecuencia esperada inferior a 5 . La frecuencia mínima esperada es 8,16. 
Medidas simétricas

\begin{tabular}{|c|c|c|c|c|c|}
\hline & & Valor & $\begin{array}{l}\text { Error tip. } \\
\text { asint. }^{a}\end{array}$ & T. aproximadab & $\begin{array}{l}\text { Sig. } \\
\text { aproximada }\end{array}$ \\
\hline Intervalo por intervalo & R de Pearson & 0,521 & 0,110 & 4,224 & $0,000^{c}$ \\
\hline Ordinal por ordinal & Correlación de Spearman & 0,521 & 0,110 & 4,224 & $0,000^{c}$ \\
\hline $\mathrm{N}^{0}$ de casos válidos & & 50 & & & \\
\hline
\end{tabular}

El p-value es menor a un $\alpha=0,05$, por lo que se rechaza la hipótesis planteada.

Existe suficiente evidencia estadística para afirmar que los factores de la cavidad bucal son determinantes en la mejora de la microfiltración de las restauraciones de las piezas dentarias con un nivel de significación de 0,05 .

Ho: La selección de los materiales de restauración no serán determinantes en la mejora de la microfiltración de las piezas dentarias.

H1: La selección de los materiales de restauración serán determinantes en la mejora de la microfiltración de las piezas dentarias.

¿Utiliza las técnicas adecuadas para insertar materiales en restauraciones grandes? ¿En cavidades complejas siempre existe el riesgo de microfiltración?

Tabla de contingencia

\begin{tabular}{|c|c|c|c|c|}
\hline \multirow[b]{2}{*}{$\%$ del total } & & \multicolumn{2}{|c|}{$\begin{array}{l}\text { ¿En cavidades complejas } \\
\text { siempre existe el riesgo de } \\
\text { microfiltración? }\end{array}$} & \multirow{2}{*}{ Total } \\
\hline & & No & Sí & \\
\hline ¿Utiliza las técnicas adecuadas & No & $22,0 \%$ & $12,0 \%$ & $34,0 \%$ \\
\hline $\begin{array}{l}\text { para insertar materiales en } \\
\text { restauraciones grandes? }\end{array}$ & Sí & $12,0 \%$ & $54,0 \%$ & $66,0 \%$ \\
\hline Total & & $34,0 \%$ & $66,0 \%$ & $100,0 \%$ \\
\hline
\end{tabular}

Pruebas de Chi-cuadrado

\begin{tabular}{|c|c|c|c|c|c|}
\hline & Valor & gl & $\begin{array}{l}\text { Sig. asintótica } \\
\text { (bilateral) }\end{array}$ & $\begin{array}{l}\text { Sig. exacta } \\
\text { (bilateral) }\end{array}$ & $\begin{array}{l}\text { Sig. exacta } \\
\text { (unilateral) }\end{array}$ \\
\hline Chi-cuadrado de Pearson & $10,822^{b}$ & 1 & 0,001 & & \\
\hline Corrección por continuidad ${ }^{a}$ & 8,848 & 1 & 0,003 & & \\
\hline Razón de verosimilitud & 10,736 & 1 & 0,001 & & \\
\hline Estadístico exacto de Fisher & & & & 0,002 & 0,002 \\
\hline Asociación lineal por lineal & 10,606 & 1 & 0,001 & & \\
\hline $\mathrm{N}^{0}$ de casos válidos & 50 & & & & \\
\hline
\end{tabular}




\begin{tabular}{|c|c|c|c|c|c|}
\hline \multicolumn{6}{|c|}{ Medidas simétricas } \\
\hline & & Valor & $\begin{array}{l}\text { Error tip. } \\
\text { asint. }^{\mathrm{a}}\end{array}$ & T. aproximada ${ }^{b}$ & $\begin{array}{c}\text { Sig. } \\
\text { aproximada }\end{array}$ \\
\hline Intervalo por intervalo & R de Pearson & 0,465 & 0,132 & 3,641 & $0,001^{\mathrm{c}}$ \\
\hline Ordinal por ordinal & Correlación de Spearman & 0,465 & 0,132 & 3,641 & $0,001^{\mathrm{c}}$ \\
\hline $\mathrm{N}^{0}$ de casos válidos & & 50 & & & \\
\hline
\end{tabular}

¿Utiliza los aditamentos o aparatos adecuados para insertar material en restauraciones complejas y disminuir la microfiltración? ¿En cavidades complejas siempre existe el riesgo de microfiltración?

Tabla de contingencia

\begin{tabular}{llccc} 
& & \multicolumn{3}{c}{ ¿En cavidades complejas } \\
siempre existe el riesgo de & \\
\% del total & & \multicolumn{2}{c}{ microfiltración? } & Total \\
\hline ¿Utiliza las aditamentos & No & $22,0 \%$ & $14,0 \%$ & $36,0 \%$ \\
$\begin{array}{l}\text { o aparatos adecuadas para } \\
\text { insertar material en }\end{array}$ & Sí & $12,0 \%$ & $52,0 \%$ & $64,0 \%$ \\
$\begin{array}{l}\text { restauraciones complejas y } \\
\text { disminuir la microfiltración? }\end{array}$ & & & & \\
Total & & $34,0 \%$ & $66,0 \%$ & $100,0 \%$ \\
\hline
\end{tabular}

Pruebas de Chi-cuadrado

\begin{tabular}{|c|c|c|c|c|c|}
\hline & Valor & $\mathrm{gl}$ & $\begin{array}{l}\text { Sig. asintótica } \\
\text { (bilateral) }\end{array}$ & $\begin{array}{l}\text { Sig. exacta } \\
\text { (bilateral) }\end{array}$ & $\begin{array}{l}\text { Sig. exacta } \\
\text { (unilateral) }\end{array}$ \\
\hline Chi-cuadrado de Pearson & $9,212^{\mathrm{b}}$ & 1 & 0,002 & & \\
\hline Corrección por continuidad ${ }^{a}$ & 7,421 & 1 & 0,006 & & \\
\hline Razón de verosimilitud & 9,162 & 1 & 0,002 & & \\
\hline Estadístico exacto de Fisher & & & & 0,004 & 0,003 \\
\hline Asociación lineal por lineal & 9,028 & 1 & 0,003 & & \\
\hline $\mathrm{N}^{0}$ de casos válidos & 50 & & & & \\
\hline
\end{tabular}

a. Calculado sólo para una tabla de 2x2.

b. 0 casillas (0\%) tienen una frecuencia esperada inferior a 5 . La frecuencia mínima esperada es 6,12 .

Medidas simétricas

\begin{tabular}{|c|c|c|c|c|c|}
\hline & & Valor & $\begin{array}{l}\text { Error tip. } \\
\text { asint. }^{a}\end{array}$ & T. aproximada & $\begin{array}{l}\text { Sig. } \\
\text { aproximada }\end{array}$ \\
\hline Intervalo por intervalo & R de Pearson & 0,429 & 0,133 & 3,293 & $0,002^{\mathrm{c}}$ \\
\hline Ordinal por ordinal & Correlación de Spearman & 0,429 & 0,133 & 3,293 & $0,002^{c}$ \\
\hline $\mathrm{N}^{0}$ de casos válidos & & 50 & & & \\
\hline
\end{tabular}


El p-value es menor a un $\alpha=0,05$, por lo que se rechaza la hipótesis planteada.

Existe suficiente evidencia estadística para afirmar que la selección de los materiales de restauración será determinante en la mejora de la microfiltración de las piezas dentarias, con un nivel de significación de 0,05 .

\section{Hipótesis especifica}

Ho: Las estructuras del prototipo no son determinantes para mejorar la microfiltración en las restauraciones de las piezas dentarias.

H1: Las estructuras del prototipo son determinantes para mejorar la microfiltración en las restauraciones de las piezas dentarias.

Tabla de contingencia. ¿Conoce usted un prototipo dental de simulador de contactos proximales que sirva para realizar estudios in vitro? ¿El prototipo que sirva para medir de simulador de contactos proximales ayudaría con la ciencia de los materiales fotopolimerizables?

\begin{tabular}{llccc} 
& & $\begin{array}{c}\text { ¿El prototipo que sirva para } \\
\text { medir de simulador de } \\
\text { contactos proximales } \\
\text { aydaría con la ciencia de los } \\
\text { materiales fotopolimerizables? }\end{array}$ & Total \\
\% del total & & No & Sí & \\
\hline $\begin{array}{l}\text { ¿Conoce usted un prototipo } \\
\text { dentral de simulador de } \\
\text { contactos proximales que } \\
\text { sirva para realizar }\end{array}$ & No & $34,0 \%$ & $4,0 \%$ & $38,0 \%$ \\
estudios in vitro? & & $10,0 \%$ & $52,0 \%$ & $62,0 \%$ \\
Total & & & & \\
\hline
\end{tabular}

\begin{tabular}{|c|c|c|c|c|c|}
\hline \multicolumn{6}{|c|}{ Pruebas de Chi-cuadrado } \\
\hline & Valor & gl & $\begin{array}{l}\text { Sig. asintótica } \\
\text { (bilateral) }\end{array}$ & $\begin{array}{l}\text { Sig. exacta } \\
\text { (bilateral) }\end{array}$ & $\begin{array}{l}\text { Sig. exacta } \\
\text { (unilateral) }\end{array}$ \\
\hline Chi-cuadrado de Pearson & $25,718^{b}$ & 1 & 0,000 & & \\
\hline Corrección por continuidad ${ }^{\mathrm{a}}$ & 22,828 & 1 & 0,000 & & \\
\hline Razón de verosimilitud & 28,414 & 1 & 0,000 & & \\
\hline Estadístico exacto de Fisher & & & & 0,000 & 0,000 \\
\hline Asociación lineal por lineal & 25,204 & 1 & 0,000 & & \\
\hline $\mathrm{N}^{0}$ de casos válidos & 50 & & & & \\
\hline
\end{tabular}




\begin{tabular}{|c|c|c|c|c|c|}
\hline \multicolumn{6}{|c|}{ Medidas simétricas } \\
\hline & & Valor & $\begin{array}{l}\text { Error tip. } \\
\text { asint. }^{a}\end{array}$ & T. aproximada ${ }^{b}$ & $\begin{array}{c}\text { Sig. } \\
\text { aproximada }\end{array}$ \\
\hline Intervalo por intervalo & R de Pearson & 0,717 & 0,098 & 7,130 & $0,000^{c}$ \\
\hline Ordinal por ordinal & Correlación de Spearman & 0,717 & 0,098 & 7,130 & $0,000^{c}$ \\
\hline $\mathrm{N}^{0}$ de casos válidos & & 50 & & & \\
\hline
\end{tabular}

El p-value es menor a un $\alpha=0,05$, por lo que se rechaza la hipótesis planteada.

Existe suficiente evidencia estadística para afirmar que las estructuras del prototipo son determinantes para mejorar la microfiltración en las restauraciones de las piezas dentarias, con un nivel de significación de 0,05 .

Ho: Los efectos de la polimerización no influyen en la microfiltración en las restauraciones de las piezas dentarias.

H1: Los efectos de la polimerización influyen en la microfiltración en las restauraciones de las piezas dentarias.

¿Sabe cómo disminuir la contracción de polimerización de estos materiales? ¿En cavidades complejas siempre existe el riesgo de microfiltración?

Tabla de contingencia.

\begin{tabular}{llccc} 
& & \multicolumn{2}{c}{$\begin{array}{c}\text { iEn cavidades complejas } \\
\text { siempre existe el riesgo de } \\
\text { microfiltración? }\end{array}$} & Total \\
\% del total & & No & Sí & \\
\hline ¿Sabe cómo disminuir la & No & $32,0 \%$ & $18,0 \%$ & $50,0 \%$ \\
$\begin{array}{l}\text { contracción de polimerización } \\
\text { en esto materiales? }\end{array}$ & Sí & $2,0 \%$ & $48,0 \%$ & $50,0 \%$ \\
Total & & & & \\
\hline
\end{tabular}

\begin{tabular}{|c|c|c|c|c|c|}
\hline \multicolumn{6}{|c|}{ Pruebas de Chi-cuadrado } \\
\hline & Valor & $\mathrm{gl}$ & $\begin{array}{l}\text { Sig. asintótica } \\
\text { (bilateral) }\end{array}$ & $\begin{array}{l}\text { Sig. exacta } \\
\text { (bilateral) }\end{array}$ & $\begin{array}{l}\text { Sig. exacta } \\
\text { (unilateral) }\end{array}$ \\
\hline Chi-cuadrado de Pearson & $20,053^{b}$ & 1 & 0,000 & & \\
\hline Corrección por continuidad ${ }^{a}$ & 17,469 & 1 & 0,000 & & \\
\hline Razón de verosimilitud & 23,035 & 1 & 0,000 & & \\
\hline Estadístico exacto de Fisher & & & & 0,000 & 0,000 \\
\hline Asociación lineal por lineal & 19,652 & 1 & 0,000 & & \\
\hline $\mathrm{N}^{0}$ de casos válidos & 50 & & & & \\
\hline
\end{tabular}




\begin{tabular}{|c|c|c|c|c|c|}
\hline \multicolumn{6}{|c|}{ Medidas simétricas } \\
\hline & & Valor & $\begin{array}{c}\text { Error tip. } \\
\text { asint. }^{\mathrm{a}}\end{array}$ & T. aproximada ${ }^{\mathrm{b}}$ & $\begin{array}{l}\text { Sig. } \\
\text { aproximada }\end{array}$ \\
\hline Intervalo por intervalo & R de Pearson & 0,633 & 0,096 & 5,669 & $0,000^{c}$ \\
\hline Ordinal por ordinal & Correlación de Spearman & 0,633 & 0,096 & 5,669 & $0,000^{c}$ \\
\hline No de casos válidos & & 50 & & & \\
\hline
\end{tabular}

a. Asumiendo la hipótesis alternativa.

b. Empleando el error típico asintótico basado en la hipótesis nula.

c. Basada en la aproximación normal.

¿Utiliza con frecuencia material restaurador fotopolimerizable en el sector posterior? ¿En cavidades complejas siempre existe el riesgo de microfiltración?

Tabla de contingencia

\begin{tabular}{|c|c|c|c|c|}
\hline \multirow[b]{2}{*}{$\%$ del total } & & \multicolumn{2}{|c|}{$\begin{array}{l}\text { ¿En cavidades complejas } \\
\text { siempre existe el riesgo de } \\
\text { microfiltración? }\end{array}$} & \multirow[t]{2}{*}{ Total } \\
\hline & & No & Sí & \\
\hline ¿Utiliza con frecuencia & No & $28,0 \%$ & $2,0 \%$ & $30,0 \%$ \\
\hline $\begin{array}{l}\text { material restaurador } \\
\text { fotopolimerizable en } \\
\text { el sector posterior? }\end{array}$ & Sí & $6,0 \%$ & $64,0 \%$ & $70,0 \%$ \\
\hline Total & & $34,0 \%$ & $66,0 \%$ & $100,0 \%$ \\
\hline
\end{tabular}

Pruebas de Chi-cuadrado

\begin{tabular}{|c|c|c|c|c|c|}
\hline & Valor & gl & $\begin{array}{l}\text { Sig. asintótica } \\
\text { (bilateral) }\end{array}$ & $\begin{array}{l}\text { Sig. exacta } \\
\text { (bilateral) }\end{array}$ & $\begin{array}{l}\text { Sig. exacta } \\
\text { (unilateral) }\end{array}$ \\
\hline Chi-cuadrado de Pearson & $33,618^{b}$ & 1 & 0,000 & & \\
\hline Corrección por continuidad ${ }^{a}$ & 29,947 & 1 & 0,000 & & \\
\hline Razón de verosimilitud & 36,280 & 1 & 0,000 & & \\
\hline Estadístico exacto de Fisher & & & & 0,000 & 0,000 \\
\hline Asociación lineal por lineal & 32,945 & 1 & 0,000 & & \\
\hline No de casos válidos & 50 & & & & \\
\hline
\end{tabular}

a. Calculado sólo para una tabla de $2 \times 2$.

b. 0 casillas $(0 \%)$ tienen una frecuencia esperada inferior a 5. La frecuencia mínima esperada es 5,10.

Medidas simétricas

\begin{tabular}{|c|c|c|c|c|c|}
\hline & & Valor & $\begin{array}{l}\text { Error tip. } \\
\text { asint. }^{a}\end{array}$ & T. aproximada ${ }^{b}$ & $\begin{array}{c}\text { Sig. } \\
\text { aproximada }\end{array}$ \\
\hline Intervalo por intervalo & R de Pearson & 0,820 & 0,085 & 9,925 & $0,000^{\mathrm{c}}$ \\
\hline Ordinal por ordinal & Correlación de Spearman & 0,820 & 0,085 & 9,925 & $0,000^{c}$ \\
\hline $\mathrm{N}^{0}$ de casos válidos & & 50 & & & \\
\hline
\end{tabular}

a. Asumiendo la hipótesis alternativa.

b. Empleando el error típico asintótico basado en la hipótesis nula.

c. Basada en la aproximación normal.

El p-value es menor a un $\alpha=0,05$, por lo que se rechaza la hipótesis planteada.

Existe suficiente evidencia estadística para afirmar que los efectos de la polimerización influyen directamente en la microfiltración en las restauraciones de las piezas dentarias, con un nivel de significación de 0,05. 


\section{RESULTADOS}

La investigación constituye una fuente inagotable de experimentación sobre el uso de tecnología de punta en la práctica odontológica en el país.

En el caso que nos ocupa, las respuestas de la gran mayoría de profesionales consultados a través de la encuesta nos permiten señalar las siguientes limitaciones:

$1^{\circ}$. Las restauraciones de piezas dentarias constituyen un desafío permanente en nuestro quehacer por la demanda existente en la población, y los resultados son positivos en cuanto se reconoce que estas quedan bien adaptadas en los pacientes.

Cuando estas restauraciones son grandes o complejas por el número de piezas, también presentan buena aceptación, lo que habla a favor de la calidad profesional de los odontólogos que trabajan en los policlínicos periféricos de la Sanidad de la Policía Nacional.

20. En el ámbito tecnológico, las restauraciones odontológicas a veces presentan algunas grietas, que animan a diagnosticar un mal pronóstico de la adaptación. Lo cierto es que los colegas señalan rápidamente que es la práctica odontológica la causa directa de esta mala adaptación, sin valorar que muchas veces la falta de tecnología e instrumentos adecuados no permite mejorar una mala adaptación.

Esta mala adaptación marginal puede desaparecer si se aplica la técnica adecuada y el uso de materiales que puedan disminuir la contracción de polimerización de dichos materiales, tanto en restauraciones grandes como pequeñas.

$3^{\circ}$. Ha quedado evidenciado que el uso de aditamentos o aparatos adecuados para insertar material en restauraciones complejas disminuye la microfiltración, incluso advirtiendo riesgos en cavidades complejas. Contrariamente a lo que se piensa, el uso de limadura de plata en restauraciones posteriores no garantiza el éxito total en este tipo de intervenciones. Es la razón por la que los odontólogos preferimos utilizar frecuentemente material restaurador fotopolimerizable en el sector posterior, además de una adecuada base para las cavidades profundas, advirtiendo la existencia de algunas dificultades para llevar la fuente de luz hacia el material fotocurado. Se recomienda entonces utilizar otros aditamentos para disminuir la distancia de la punta de la fuente de luz al material fotocurado.

$4^{\circ}$. De otro lado, la mayoría de odontólogos consultados asevera conocer un prototipo dental de simulador de contactos proximales para realizar estudios in vitro, cuyo prototipo sirve para medir también la simulación de contactos proximales y así ayudar a la práctica odontológica con el uso científico de los materiales fotopolimerizables. El único problema que se advierte es el alto costo requerido para diseñar un prototipo con este propósito.

\section{CONCLUSIONES}

\section{Conclusión general}

Existe suficiente evidencia científica para afirmar que el prototipo simulador dental mejorará las microfiltraciones en las restauraciones de las piezas dentarias de los pacientes que acuden a los consultorios externos de los tres policlínicos periféricos de la Sanidad de la Policía Nacional del Perú en Lima Metropolitana.

\section{Conclusiones parciales}

1. Existe suficiente evidencia estadística para afirmar que los factores de la cavidad bucal 
son determinantes en la mejora de la microfiltración de las restauraciones de las piezas dentarias de los pacientes que acuden a los policlínicos periféricos de la Sanidad de la Policía Nacional del Perú.

2. Existe suficiente evidencia estadística para afirmar que la selección de los materiales de restauraciones es determinante en la mejora de la microfiltración de las piezas dentarias de los pacientes que acuden a los policlínicos periféricos de la Sanidad de la Policía Nacional del Perú.

3. Existe suficiente evidencia estadística para afirmar que las estructuras del prototipo de simulador dental son determinantes para mejorar la microfiltración en las restauraciones de las piezas dentarias de los pacientes que acuden a los policlínicos periféricos de la Sanidad de la Policía Nacional del Perú.

4. Existe suficiente evidencia estadística para afirmar que los efectos de la polimerización influyen directamente en la microfiltración en las restauraciones de las piezas dentarias de los pacientes que acuden a los policlínicos periféricos de la Sanidad de la Policía Nacional del Perú.

\section{RECOMENDACIONES}

\section{Recomendaciones parciales}

1. Las Facultades o Carreras Profesionales de Odontología o Estomatología debieran organizar cursos de actualización dirigidos a sus agremiados sobre los últimos avances de la tecnología utilizada en cirugía dental, como es el uso del simulador dental para mejorar la microfiltración en las restauraciones de las piezas dentarias.
2. El Colegio Odontológico del Perú debiera promocionar a través de su revista especializada la experimentación continua y la difusión masiva de las últimas investigaciones que se vienen realizando en las universidades del sistema nacional.

3. La Sanidad de la Policía Nacional del Perú debiera implementar en la red de policlínicos periféricos que atienden en Lima Metropolitana la utilización de nueva tecnología en operatoria dental, como es el prototipo de simulador dental para mejorar la microfiltración en las restauraciones de las piezas dentarias de los nuevos pacientes que acuden a sus consultorios externos.

\section{REFERENCIAS BIBLIOGRÁFICAS}

1. Lutz F, Phillips RW, Rouler JF, Sercos JC. In vivo and in vitro of potencial posterior composites, J Dent Res 1984; 63: 914-920.

2. Rueggeberg FA, Caughman WF, Curtis JW, Jr., Dawis HC. Factors affecting cure at depth within light-activated resin composites. Am Jden 1993; 6: 91-95.

3. Kilian RJ. The application of photochemistry to dental material, in Gebelein CG, Koblirz FK. Biomedical and dental application of polymers. New York: Pleniun Press;1981: 411-416.

4. Pearson CJ, Longmn CM. Water Sorption and solubility of resin based materials following inadecuate polymerization by a visiblelight curing system. J Oral Rehab 1989; 16: 57-61.

5. Curtis JW, Rueggeberg FA, et al. Eficiencia del fotocurado con puntaturbo. J Clínica en Odont 1996/1997; 12(3): 19-27.

6. Pires JA. Effects of curing tip distance on light intensity and compose resin micro hardness. Quintessence Int. 1993, 24 (7): 517-521. 
7. Marra LM: An historical review of full coverage of the natural dentition.NY State Dent $\mathrm{J}: 36: 147.1970$.

8. Smith, D. Spreafico: Adhesivo metal free restauations. Quintessence International Chicago, 1997.

9. Bowen, R.L : Dental Filling Material comprising vinil silane trated fused silica and a binder consisting of the reaction product of bisphenol and glycitil acrilate. US. Patent No 3,066, 112, 1962.

10. Ott, G Composites filling materials-ivoclarvivadent. Report. No.5. 1990: 3-5.

11. Ferracane, JL. Current trnds in Dental Composites. Crit Rev. Oral Biol Med. 1995; 6(4): 302-318.

12. Phillips, RW. Composite Resin System (comments) Annals Academy of Medicine. 1996; 15(3): 438.

13. Nadarajah, V.et al. Local inflamatory effects of Composte Resins. Compendium. 1997; 18 (4): 367-374.

14. Bayne, S.C et. Al Update on dental composite restorations. J. Amer. Dent. Assoc. 1994; 125: 687-701.

15. Leinfelder, K.F Posterior Composites. State of the art, clinical applications. Dent. N. Amer. 1993; 37(3): 411-418.
16. Adep Report, Alberts, HF, editor. Direct Composite Restoratives. 1991; 2(4):53-65.

17. R.W.Bryant. Resinas de composite 200;IX. 95-97

18. Chung (1990) The relationship between composition and properties of posterior resin composites. Journal Dental Research. 69 (3): $852-856$.

19. Macorra Garcia and others (2000) Polymerization contraction of composite resin restorative materials. Sociedad Española de Odontologia Conservadora. Volumen 2. $\mathrm{N}^{\circ}$ $1: 24-35$.

20. Buschlicher and others (1997) Effect of composite type, light intensity, configuration and lase polymerization. American Journal Dental 10: 88 - 96 .

21. Versluis and Others (1998) Do Dental composite resins always shrink towards the light? JournalDental Research. 77(6): 1435 -1445 .

22. Asmussen E. (1998) Direction of shrinkage of light curing resin composites. Journal Dental Research. 77 Abstr. no 212.

23. Hansen (1982) Visible light cured composite resins. Scandinava. JournalDental research 90: $329-335$. 